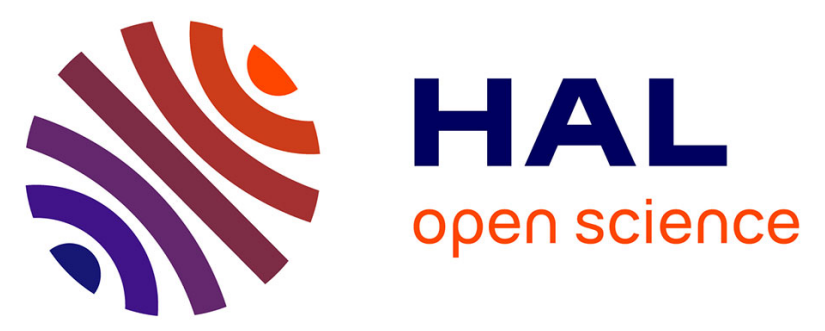

\title{
Efficience et obligation de résultat: quelles leçons tirer de la politique points noirs de l'agence Seine Normandie menée de 1987 à 1991 ?
}

Gabrielle Bouleau, Y. Lunet de La Jonquière

\section{- To cite this version:}

Gabrielle Bouleau, Y. Lunet de La Jonquière. Efficience et obligation de résultat: quelles leçons tirer de la politique points noirs de l'agence Seine Normandie menée de 1987 à 1991 ?. La Houille Blanche - Revue internationale de l'eau, 2007, 3, p. 58 - p. 63. 10.1051/lhb:2007036 . hal-00453818

\section{HAL Id: hal-00453818 \\ https://hal.science/hal-00453818}

Submitted on 5 Feb 2010

HAL is a multi-disciplinary open access archive for the deposit and dissemination of scientific research documents, whether they are published or not. The documents may come from teaching and research institutions in France or abroad, or from public or private research centers.
L'archive ouverte pluridisciplinaire HAL, est destinée au dépôt et à la diffusion de documents scientifiques de niveau recherche, publiés ou non, émanant des établissements d'enseignement et de recherche français ou étrangers, des laboratoires publics ou privés. 


\title{
Efficience et obligation de résultats : quelles leçons tirer de la politique points noirs de l'agence Seine Normandie menée de 1987 à 1991 ?
}

\author{
Most cost-effective options: what lessons can be learned from the black \\ spots strategy of "Agence de l'Eau Seine-Normandie" in 1987-1991?
}

\section{GABRIELLE BOULEAU, YVES LUNET DE LAJONQUIERE}

UMR G-EAU, Cemagref

361, rue J.F. Breton, BP 5095, 34196 Montpellier Cedex 5

Tél. : 33 + (0)4 670463 51, Fax : 33 + (0)4 671664 40, e-mail : gabrielle.bouleau@cemagref.fr

$D$ uring the period 1987-1991, the French Water Agency of Seine-Normandy basin experimented with a "black spots" strategy in order to restore aquatic sites of very poor quality and of great interest for usages. The Agency assessed the required investments, increased subsidies and communication efforts there. We tried to draw lessons from this policy with an ex-post appraisal of its results.Data collection was an important part of our work given the absence of a Geographical Information System in the Agency. We built a database of 58 "black spots" which can be considered as well informed case studies with comprehensive financial data and water quality monitoring. We propose indicators to assess the black-spots strategy. We show that, given the general basinwide quality getting worse, the strategy was effective and efficient. Without any data concerning usage recovery, the weight of the different criteria such as length of restored river or number of restored spots remains subjective. Different variables likely to explain success and failure are tested. Variability of discharges explains partly differences of results. We also enlighten a regional office effect.Such a site-oriented approach illustrates how different causes combine with each other to impact the environment. To assess consistency and effectiveness of any strategy, one should previously list all possible causes of degradation and possible remediation site by site to make them comparable and to understand properly their evolution. Moreover monitoring options may deeply affect scoring results.

I INTRODUCTION : LA DIRECTIVE CADRE EUROPEENNE SUR L'EAU QUESTIONNE LA POLITIQUE POINTS NOIRS SOUS UN ANGLE NOUVEAU

Les Agences de l'Eau en France sont nées d'une première loi sur l'eau instituant les agences financières de bassin (loi sur le régime des eaux du 16 décembre 1964). La loi sur l'eau de 1992 a ensuite renommé les agences de l'eau et élargi leurs compétences vers une gestion planifiée des ressources et des milieux aquatiques.

Des années 60 aux années 90, l'effort de dépollution pour respecter les normes (eaux de baignade, eaux potabilisables, eaux résiduaires urbaines) et les conventions internationales (OSPAR, Helsinki,...) était tel qu'il y avait des investissements à faire à peu près sur toutes les eaux. Si les montants des programmes quinquennaux n'étaient pas limitants, il n'y avait pas de raison d'établir des priorités. Le critère de choix était souvent l'existence d'un maître d'ouvrage motivé pour réaliser des investissements que de toute façon il fallait faire.

Le succès des agences de l'eau depuis 1964 est d'avoir permis à des industriels, des éleveurs et des collectivités de financer leurs investissements de dépollution sans recourir à des emprunts à taux élevés. Le nombre de services d'eau ou d'assainissement en France est d'environ 35 000, il s'agit donc en moyenne de petits services [1] qui ont un besoin permanent de péréquation inter-services pour lisser les décaissements correspondants à leurs investissements [2]. Jusqu'à récemment, les six agences de l'eau assumaient, grâce à leurs redevances pour pollution et pour prélèvement reversées sous forme de subventions, ce rôle de péréquation dans l'espace et le temps, comme des mutuelles [3]. Depuis 1992, les agences ont à élaborer conjointement avec les Directions Régionales de l'Environnement (DIREN) les Schémas Directeurs d'Aménagement et de Gestion des Eaux (SDAGE) qui définissent les grandes orientations au niveau du bassin et qui doivent être mis à jour avec la Directive Cadre Européenne sur l'eau (DCE). Les DIREN sont chargées du suivi de la qualité de l'eau et d'harmoniser les politiques publiques en matière d'eau, les agences sont le principal instrument financier de la politique publique de l'eau et notamment de la dépollution $[4 ; 5]$. Dotées de programmes quinquennaux conséquents (près de 1,6 milliard d'Euros par an pendant la période 2003-2006) les agences de l'eau sont des acteurs incontournables pour définir et mettre en œuvre le programme de mesures exigé par la DCE.

Cette directive adoptée en 2000 exige, en effet, que les Etats membres établissent un programme de mesures tous les six ans pour restaurer la qualité de toutes les masses d'eaux. Ces mesures comprendront des limitations d'usages, des incitations et des investissements et devront être adaptées à 
chaque type d'eau. Pour chaque masse d'eau, l'écart entre l'état actuel et l'objectif devra être évalué et les mesures devront être dimensionnées en conséquence. C'est pourquoi certains observateurs considèrent que la directive cadre va induire un changement très important dans la politique française de l'eau puisque la sélectivité des aides n'a jamais été pratiquée auparavant $[6 ; 7]$.

Est-ce que le cinquième programme d'intervention de l'Agence de l'Eau Seine Normandie (1987-1991) ne serait pas une exception? Etant donné la rigueur budgétaire de l'époque, cette Agence affichait dans son programme une stratégie visant à concentrer les investissements sur des points noirs : des tronçons de rivières, des nappes et des bordures littorales où la qualité de l'eau menaçait les usages existants. Afin d'encourager les maîtres d'ouvrage (principalement des collectivités et des industriels) à réduire rapidement leurs impacts, l'Agence de l'Eau Seine Normandie (AESN) majorait ses taux d'aides aux pollueurs de points noirs (subventions à la dépollution passant en général de 30 à $40 \%$ ) sans modifier les taux de redevances sur les zones correspondantes. 123 "points noirs" furent sélectionnés correspondants à 98 tronçons de rivière $(1819 \mathrm{Km}), 11$ aquifères et 16 portions de côte (voir Figure 1).

Un bilan annuel de ces points noirs était présenté au Conseil d'Administration de l'Agence. Pour chaque point, étaient indiqués la localisation de la ressource en eau concernée, les paramètres déclassants, les causes possibles de la dégradation avec le nom et la commune des éventuels pollueurs connus et l'avancement de la résorption depuis le diagnostic jusqu'à l'achèvement des travaux nécessaires (voir Tableau 1). Ce double suivi de l'évolution de la qualité de l'eau d'un site et des investissements réalisés sur ce site n'a jamais été réalisé à un autre moment par les agences. Il s'agit donc d'un échantillon de sites pour lesquels une mesure de l'impact relatif de l'action de l'Agence peut être fait (partout ailleurs et jusqu'à aujourd'hui l'absence de géoréférencement des données de l'Agence ne permet pas de relier les subventions aux milieux aquatiques concernés).

Ces données ont conduit l'Agence de l'Eau Seine Normandie à se demander si des enseignements de ce programme ne pouvaient pas être tirés pour éclairer les difficultés éventuelles liées à la sélectivité et à l'obligation de résultat imposés par la DCE. Deux études ont été menées à titre exploratoire. La première avait mis en avant les enjeux financiers et les difficultés politiques liées à la sélectivité qui remet en cause le fonctionnement mutualiste des agences [8]. La deuxième avait pour but de définir une méthode de tri et d'analyse des données points noirs. Elle avait permis de tracer les investissements sur 27 points noirs dont l'évolution en qualité était connue [9]. La présente étude s'appuie

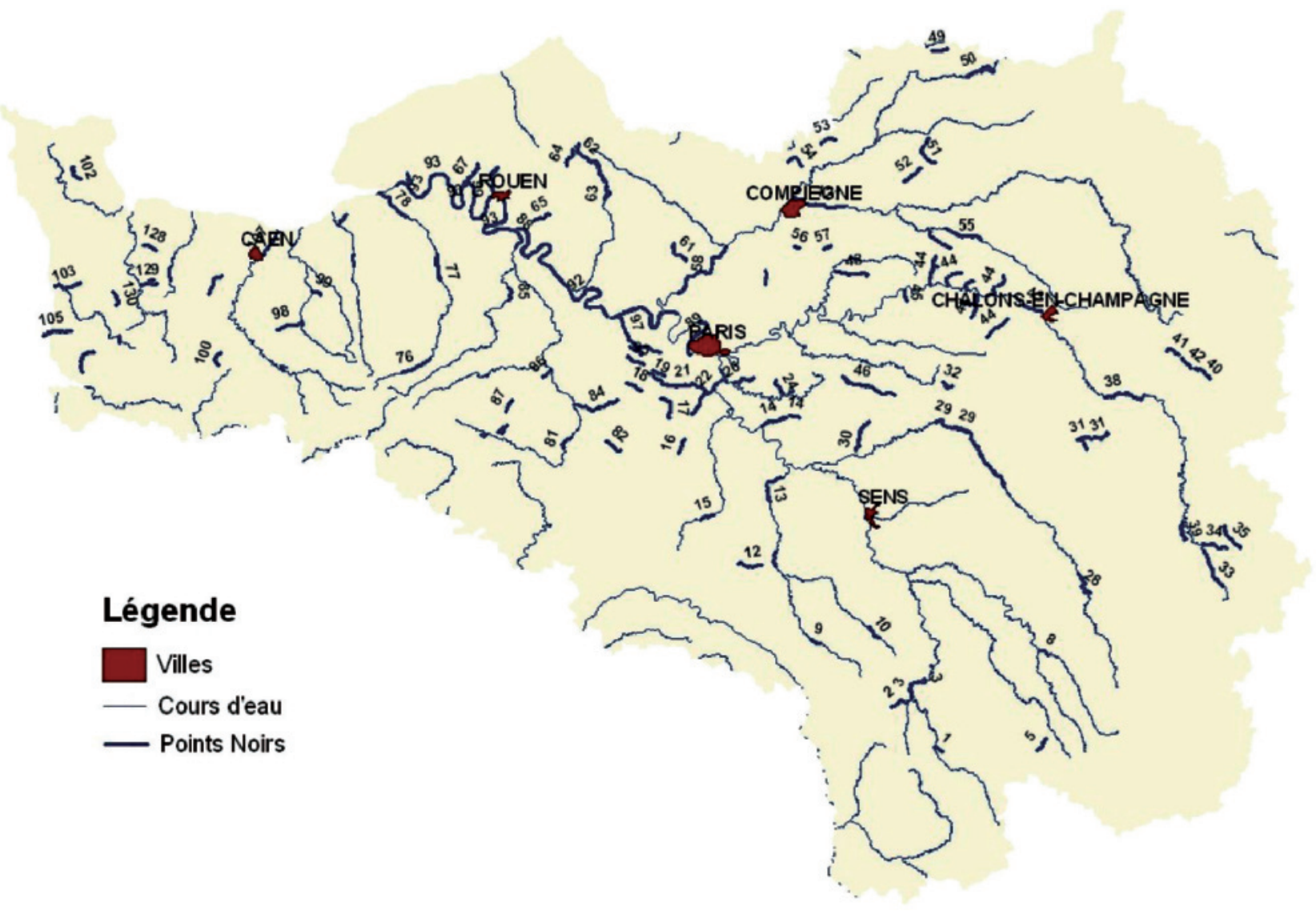

Figure 1 : Localisation géographique des 123 points noirs du bassin Seine Normandie pendant le cinquième programme 1987-1991. 
Tableau 1. Exemple d'information disponible dans le bilan annuel des 123 points noirs pour le conseil d'administration de l'AESN.

\begin{tabular}{|l|c|}
\hline Numéro du point noir & 1 \\
\hline Aquifère ou exutoire & Anguison \\
\hline Localisation précise & Coral de Corbigny \\
\hline Pollueurs potentiels & $1 \mathrm{~B}$ \\
\hline Objectifs de qualité & \multicolumn{1}{c|}{0.27} \\
\hline Débit moyen $\left(\mathrm{m}^{3} / \mathrm{s}\right)$ & $\begin{array}{c}\text { Qualité } 2 \text { en } 1982, \text { qualité } 3 \text { en } 1983 \text { en conditions moyennes de débit. Paramètres } \\
\text { déclassants : NH4, DBO, DCO et surtout Indice biotique. Pas d'évolution notable. }\end{array}$ \\
\hline $\begin{array}{l}\text { Information sur la Détérioration } \\
\text { de la qualité }\end{array}$ & Diagnostic du réseau d'eaux usées \\
\hline Diagnostic & I (I : aucun avancement, V : résorption) \\
\hline Avancement & \\
\hline
\end{tabular}

sur ces deux premiers travaux et généralise leurs conclusions à un échantillon de points noirs plus important obtenu en affinant la procédure de tri.

\section{M METHODOLOGIE UTILISEE POUR COUPLER LA QUALITE DE L'EAU AUX DONNEES FINANCIERES}

Nous avons cherché à construire une base de données contenant tous les points noirs et les subventions correspondantes ainsi que l'évolution de la qualité de l'eau avant et après le programme. Cette construction a nécessité un tri des subventions accordées pendant le cinquième programme pour ne retenir que celles qui correspondent à un point noir (information non disponible d'emblée). Puis il a fallu coupler ces points noirs financés à une évolution de la qualité de l'eau locale.

Aujourd'hui les bases de données des financements accordés par les agences ne mentionnent que le siège du bénéficiaire. Pour optimiser les mesures de restauration et répondre à l'obligation de résultat sans refaire à chaque fois ce tri fastidieux où l'on doit abandonner des points faute d'information, il sera nécessaire de disposer d'une base de données des investissements qui précise quels sont les milieux aquatiques concernés. Il sera aussi important de pouvoir se référer à des mesures de qualité de l'eau qui soient comparables. Ce sera probablement l'une des tâches du nouvel Office National de l'Eau et des Milieux Aquatiques créé par la loi du 30 décembre 2006.

\section{II.1 CONSTRUCTION D'UNE BASE FINANCIÈRE RELATIVE AUX POINTS NOIRS}

Notre sélection repose sur la cohérence entre la nature des travaux de dépollution aidés par l'agence au cinquième programme et le diagnostic des travaux nécessaires pour résorber les points noirs tels que mentionnés dans le bilan annuel de 1985 ou 1990. Cette étape nous a permis de construire une base de données de 1196 opérations financées relatives à 88 points noirs. Nous avons appelé cette base « les points noirs financés »
Ce faisant, nous avons récupéré des opérations qui peuvent également relever à la fois d'un point noir et d'une autre stratégie car certaines opérations sont éligibles à plusieurs critères. Une politique "Seine Propre» et une politique "contrat d'agglomération » ont été menées à la même époque et ont donné lieu également à des majorations de taux de financement. Nous avons identifié spécifiquement les opérations Seine propre. Les contrats d'agglomération ont été assimilés aux points noirs.

Nous avons aussi récupéré des travaux jugés nécessaires dans le bilan annuel des points noirs mais qui n'ont pas bénéficié d'aides majorées. Pour certaines d'entre elles nous avons pu établir que ceci venait du fait que l'aide classique de l'agence ajoutée à celle des autres partenaires permettait déjà au maître d'ouvrage d'atteindre $80 \%$ de subvention. Nous avons donc conservé ces opérations qui n'entraînent pas de surcoût comptable pour l'agence mais qui ont dû bénéficier du même effort de communication que les autres opérations points noirs.

\section{II.2 COUPLAGE AVEC LES DONNÉES DE QUALITÉ DES EAUX}

L'étape suivante a consisté à coupler la base "points noirs financés " avec les données de qualité du milieu. Nous nous sommes restreints aux eaux superficielles et douces qui représentaient la majorité des points noirs.

Les neuf points noirs superposés au programme Seine Propre sont relatifs aux deux stations Valenton et Achères et à une opération de réduction des toxiques sur l'ensemble du cours de la Seine en aval de Paris. Les tronçons de fleuve concernés ne peuvent pas être étudiés séparément car les effets cumulatifs des pollutions sont importants. Nous avons donc choisi d'isoler ce secteur du reste de notre échantillon pour le traiter à part.

Pour les données relatives à la qualité des cours d'eau, nous avons utilisé les données corrigées par des experts des DIREN sous forme de carte de qualité, construites à partir de la grille de 1971 basée sur cinq paramètres (oxygène dissous, $\%$ de saturation en oxygène, DBO5, DCO, NH4+) et établies sur plusieurs années (cinq à sept). Le principe de 
Tableau 2. Grille de qualité de 1971 utilisée pour les cartes de qualité de 1974 à 1995.

\begin{tabular}{|l|c|c|c|c|c|}
\hline & Excellente & Bonne & Passable & Médiocre & Hors classe \\
\cline { 2 - 6 } & $\mathbf{1 A}$ & $\mathbf{1 B}$ & $\mathbf{2}$ & $\mathbf{3}$ & HC \\
\hline Oxygène dissous en mg O2/1 & $>7$ & 7 à 5 & 5 à 3 & 3 à 1,5 & $<1,5$ \\
\hline$\%$ de saturation en Oxygène & $>90$ & 90 à 70 & 70 à 50 & 50 à 20 & $<20$ \\
\hline DBO5 en mg O2/1 & $<3$ & 3 à 5 & 5 à 10 & 10 à 25 & $>25$ \\
\hline DCO mg O2/1 & $<20$ & 20 à 25 & 25 à 40 & 40 à 80 & $>80$ \\
\hline Ammonium mg NH4+/1 & $<0,1$ & 0,1 à 0,5 & 0,5 à 2 & 2 à 8 & $>8$ \\
\hline
\end{tabular}

cette grille est d'attribuer une note de qualité correspondant à la classe de qualité du paramètre le plus mauvais (Voir Tableau 2). Cette méthode utilise une mesure non linéaire de la qualité. Bien qu'improbable, il est possible qu'une grande partie du linéaire se trouve en limite inférieure d'une classe de qualité. L'amélioration serait donc invisible alors que la détérioration amènerait un changement de classe de qualité. Mais cet effet peut jouer en sens inverse en surestimant les améliorations. Nous pensons que cet effet est lissé sur l'ensemble des points.

Comme nous le verrons dans les résultats, ces données bien que très agrégées permettent déjà des conclusions intéressantes. Nous avons choisi de travailler à partir des cartes de 1977-1984 et de 1991-1995. Le bilan des points noirs au conseil d'administration de 1985 (avant le démarrage du programme) montre une grande cohérence avec la carte de qualité 1977-1984.

Le facteur le plus limitant pour notre étude a été l'abandon de nombreuses stations de mesures entre 1989 et 1991 . Nous avons donc construit une base de données plus réduite « points noirs financés et suivis en qualité » (voir Figure 2).

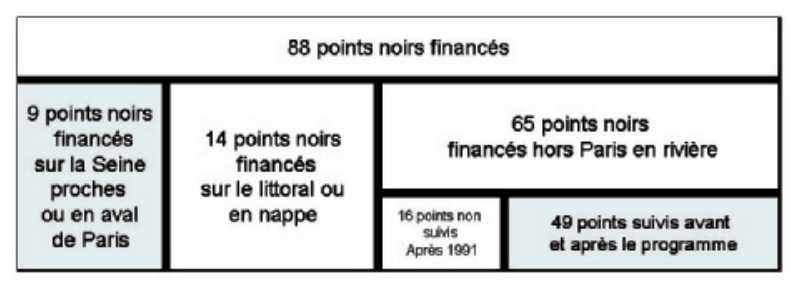

Figure 2 : Segmentation de l'information sur les points noirs. Les ensembles grisés correspondent à ceux pour lesquels il existe une information sur l'évolution de la qualité. Les montants sont des subventions effectivement payées exprimées en millions d'Euros.

\section{II.3 Analyse statistique des bases de données}

Deux types d'analyses ont été menés sur ces données : une analyse du niveau de pollution des points étudiés et une analyse comparée de l'évolution de la qualité des points noirs par rapport à l'ensemble du bassin en proposant des facteurs explicatifs de cette évolution.
Nous avons étudié la représentativité des 49 « points noirs financés suivis en qualité » par rapport à tous les points noirs et par rapports à toutes les opérations financées en dépollution. Nous avons regardé si ces 49 points se différenciaient par leur localisation régionale, par la nature des travaux, par la nature des bénéficiaires, par le débit des cours d'eau concernés, par l'année de programmation des opérations ou de l'importance financière des projets. Nous avons également mené ce travail sur les 9 points assimilés au programme Seine Propre.

Puis nous avons comparé l'évolution de la qualité de l'ensemble du bassin à celles des 49 points noirs hors Paris et des 9 points assimilables à Seine Propre. Ceci permet de distinguer des points noirs qui ont pu être résorbés des autres et de proposer des explications sur les causes des échecs ou de l'absence de résultats visibles avec la méthode retenue.

\section{III $\square$ RESULTATS}

\section{III.1 Représentativité des données traitées}

Nous avons retrouvé tous les points noirs financés, mais le suivi en qualité n'est pas possible pour tous du fait de la perte de points de mesure.

Le travail antérieur mené sur l'enjeu de la concentration financière sur les points noirs [Priet 2004] avait établi que les points noirs avaient bénéficié de 250 millions d'Euros d'aides (y compris Seine Propre) sur 400 millions d'Euros investis en dépollution entre 1987 et 1990 (Conseils d'administration de 1987 à 1990). Nous retrouvons exactement ces chiffres (250 millions d'Euros hors désengagements, 218 millions d'Euros en tenant compte des désengagements) à partir de notre base "points noirs financés » qui peut donc être considérée comme exhaustive de 1987 à 90 . Les réunions du conseil d'administration en 1991 n'ayant pas de bilan points noirs, nos données ne sont pas comparables pour cette année-là.

La comparaison des 49 "points financés et suivis en qualité » par rapport aux 65 points noirs hors Paris montre quelques biais. Ces 49 points noirs représentent un montant d'aides de 53 millions d'Euros soit $81 \%$ des investissements points noirs hors Paris. Les 16 points noirs qui n'ont pas pu être suivis en qualité hors Paris sont majoritairement situés sur des petits cours d'eau (13 d'entre eux). C'est en effet le petit chevelu de débit moyen inférieur à $0,1 \mathrm{~m}^{3} / \mathrm{s}$ qui a été 
le plus affecté par l'abandon des stations de mesures après 1991. Il faut également noter que notre échantillon de 49 points noirs représente mal la politique points noirs pour le secteur agro-alimentaire (nous ne retrouvons que $30 \%$ des investissements), pour la région de Caen ( $32 \%$ des investissements) et pour la région Ile de France hors Seine Propre (52\% des investissements).

\section{III.2 Evolution générale des points noirs financés par rapport à l'ensemble du bassin Seine Normandie}

En moyenne pondérée par leur linéaire, l'évolution de la qualité des points noirs assimilables à Seine Propre est significativement positive $(+0,16)$, l'évolution de la qualité des points noirs hors Seine Propre est un peu moindre $(+0,07)$ mais reste supérieure à l'évolution moyenne de tout le bassin $(-0,04)$. Rappelons l'abandon de points de mesure sur des petits cours d'eau qui étaient en général également de bonne qualité et dont on ne connaît pas l'évolution.

Cette évolution moyenne peut être affinée en tenant compte de la situation initiale de chaque tronçon et de ce que représente le linéaire de points noirs dans chaque classe de qualité hors Seine Propre.

Lévolution des points de qualité hors classe et médiocre correspond à celle de l'ensemble du bassin, la qualité s'améliore d'au moins une classe pour $30 \%$ du linéaire. Pour les points de qualité passable la situation n'évolue pas nettement. Pour les points de qualité bonne, celle-ci semble se dégrader. Que sont ces points noirs dont la qualité était jugée bonne d'après la carte de 1977-84 ? L'analyse fine des points noirs considérés montre qu'il s'agit surtout de quelques tronçons qui jouxtent des tronçons beaucoup plus dégradés et qui ont été assimilés au point noir adjacent probablement parce que l'agence jugeait que la pollution pouvait les toucher également. Pour d'autres, il s'agit de tronçons dont la qualité sur la carte est surévaluée par une mesure de la qualité faite en 1983, année très pluvieuse et peu représentative pour ces points dont le débit est en général très faible. Enfin certains points ont été classés en points noirs pour des dégradations dont la grille de qualité de 1971 ne rend pas compte (pollution aux métaux).

Ce premier bilan peut être interprété en termes d'efficacité et d'efficience (voir Tableau 3). L'amélioration de la qualité peut être ramenée à des kilomètres de rivières gagnant une classe de qualité. L'ensemble du bassin perd en moyenne une classe de qualité sur $452 \mathrm{Km}$, Seine Propre gagne en moyenne une classe de qualité sur $84 \mathrm{~km}$ et les autres points noirs, une classe de qualité sur $63 \mathrm{Km}$. Si l'on s'intéresse à la pollution éliminée, le gain en classe de qualité est de $+0,20$ sur Seine Propre et $+0,11$ sur les autres points noirs financés.

Ramené au surcoût induit par les aides majorées sur ces points, ce gain montre que l'amélioration d'une classe de qualité sur Seine Propre a coûté en moyenne deux fois plus cher que sur les autres points noirs.

\section{III.3 Analyse des facteurs d'efficience}

Nous avons testé différentes typologies des 49 points noirs financés pour essayer d'en déduire des facteurs expliquant la variation d'efficience. Deux facteurs semblent significatifs dans les variations d'efficience.

Le premier est la gamme de débit moyen. Les débits très faibles $\left(<0,1 \mathrm{~m}^{3} / \mathrm{s}\right)$ ne s'améliorent guère en qualité. On peut penser qu'il s'agit d'exutoires dont les effluents constituent une bonne partie du débit. La diminution éventuelle de la quantité d'effluent ne change guère les concentrations, les conditions climatiques en revanche peuvent peser beaucoup sur les mesures de qualité. Les très gros débits $\left(>10 \mathrm{~m}^{3} / \mathrm{s}\right)$ n'évoluent pas non plus favorablement en moyenne. Pour ces derniers on peut penser que les causes de dégradation sont dispersées sur un grand bassin et que leur résorption demande plus de temps.

Le cas particulier des points noirs superposés au programme Seine Propre peut aussi s'expliquer par la gamme de débit. La Seine a en effet un débit très faible pour diluer la pollution de l'agglomération parisienne. L'amélioration de qualité en aval de Paris demande donc des investissements importants.

Le deuxième est la direction de secteur qui a été chargée $\mathrm{du}$ dossier. Les directions de Caen, Sens et Rouen et la direction sectorielle chargée de la chimie et de la métallurgie ont en moyenne de bons résultats sur ces points noirs. Pour certaines d'entre elles, ces bons résultats peuvent s'expliquer par la part importante que les points noirs ont représentée dans l'ensemble des aides à la dépollution qu'elles ont eu à gérer (75\% pour Rouen, $54 \%$ pour Caen). Ces directions ont probablement eu les moyens d'affecter du temps spécifiquement aux points noirs pour un effort de sensibilisation des partenaires de l'agence. Mais cette condition n'est ni

Tableau 3. Mesures d'efficacité et d'efficience du programme Seine Propre et des Points Noirs hors Seine Propre.

\begin{tabular}{|l|c|c|c|c|c|c|c|}
\hline \multicolumn{1}{|c|}{$\begin{array}{c}\text { Tronçons } \\
\text { considérés }\end{array}$} & $\begin{array}{c}\text { Linéaire } \\
\text { concerné } \\
\text { en } \mathbf{K m} \\
\mathbf{( 1 )}\end{array}$ & $\begin{array}{c}\text { Total } \\
\text { investi } \\
\text { sur ces } \\
\text { tronçons }\end{array}$ & $\begin{array}{c}\text { Surcoût } \\
\text { lié à la } \\
\text { majoration } \\
\mathbf{( 2 )}\end{array}$ & $\begin{array}{c}\text { Gain moyen } \\
\text { en classe de } \\
\text { qualité } \\
\mathbf{( 3 )}\end{array}$ & $\begin{array}{c}\text { Effet absolu } \\
\mathbf{( 1 ) * ( 3 )} \\
\text { en équivalent Km } \\
\text { gagnant une classe }\end{array}$ & $\begin{array}{c}\text { Efficacité } \\
\text { relative } \\
\mathbf{( 3 )}+\mathbf{0 , 0 4}\end{array}$ & $\begin{array}{c}\text { Efficience } \\
\text { relative }= \\
\text { Efficacité } \\
\text { relative / surcoût }\end{array}$ \\
\hline Bassin entier & 11300 & $561 \mathrm{M} €$ & $47 \mathrm{M} €$ & $-0,04$ & -452 & & +84 \\
\hline $\begin{array}{l}\text { 9 Points noirs } \\
\text { Seine Propre }\end{array}$ & 526 & $193 \mathrm{M} €$ & $36 \mathrm{M} €$ & $+0,16$ & $+0,20$ & $+0,006 / \mathrm{M} €$ \\
\hline $\begin{array}{l}\text { 49 Points noirs } \\
\text { hors Seine Propre }\end{array}$ & 894 & $65 \mathrm{M} €$ & $11 \mathrm{M} €$ & $+0,07$ & +63 & $+0,11$ & $+0,011 / \mathrm{M} €$ \\
\hline
\end{tabular}


nécessaire (10\% pour la Chimie) ni suffisante ( $81 \%$ pour Châlons), d'autant que l'analyse statistique montre que le facteur " direction de secteur» explique peu la variabilité sur l'évolution de qualité et que l'écart type autour des moyennes par direction de secteur est élevé.

\section{DISCUSSION : LE PARTAGE DES RESPONSABILITES}

Dans toute cette analyse, nous n'avons pas tenu compte de la multiplicité des acteurs dans le domaine de l'eau. Pour qu'un point noir se résorbe, il faut toute une séquence d'actions. L'interruption à un moment donné de la chaîne d'action entraîne une absence de résultat. Pour autant, l'agence n'a pas forcément failli à sa mission. Il y a lieu de distinguer les responsabilités pour satisfaire l'obligation de résultats. Cette distinction des responsabilités n'est pas faite dans les bilans annuels d'avancement de la résorption des points noirs faits pour le compte des conseils d'administration.

Le modèle d'action de l'agence est linéaire. Il s'agit d'identifier les sources de pollution, convaincre un maitre d'ouvrage d'investir dans la dépollution, subventionner les travaux à mesure qu'ils se réalisent et observer l'amélioration de la qualité.

Les exemples de points noirs que nous avons étudiés mettent en défaut cette vision linéaire. Certains points noirs se résorbent par déplacement de l'activité polluante (fermeture d'une usine pour des raisons économiques ou du fait de la sensibilisation faite par l'agence ou la police de l'eau). L'agence peut donc parfois obtenir des résultats sans subventionner. Certains points noirs sont le fait de pollution diffuse sans maîtrise d'ouvrage. L'étape « convaincre un maitre d'ouvrage » est une impasse et il y aurait probablement lieu d'envisager d'autres modalités d'action. Certaines actions sont substituables à d'autres. Enfin les mesures de la qualité et le réseau de mesure influencent beaucoup la visibilité de l'impact.

Tout se passe comme si l'agence assumait les échecs qui incombent à d'autres. Cette représentation ne tient pas compte des responsabilités extérieures à l'agence et d'un certain nombre d'alternatives possibles dans l'arbre de décision.

\section{$\mathrm{V} \square$ CONCLUSION}

La politique points noirs menée par l'Agence Seine Normandie entre 1987 et 1991 n'a pas été poursuivie parce qu'elle risquait d'entraîner un effet d'aubaine pour les mâ̂tres d'ouvrage responsables des pollutions les plus importantes en leur accordant des subventions majorées. L'effort supplémentaire consenti sur les points noirs a été jugé inéquitable sur le long terme. Mais à court terme, cette différenciation provisoire est riche d'enseignements pour l'évaluation. En effet, en distinguant deux types d'opérations, elle permet de mesurer l'efficience des aides de l'agence. Lévaluation montre la difficulté de résorber les pollutions pour les très petits et très gros débits. Elle montre également le coût plus élevé de la dépollution dans la région centrale d'Ile de France.

Ses enseignements sont aussi méthodologiques. En essayant de quantifier l'efficience de cette politique on se rend compte que cette mesure est sensible à la méthode utilisée pour agréger les données relatives à différents paramètres de qualité. La méthode en vigueur dans les années 70 à 90 , celle de la grille de qualité de 1971 comporte un fort effet de seuil entre classe qui ne permet pas de dire de façon sûre si la qualité s'est améliorée ou dégradée sur l'ensemble du bassin avant et après le programme.

Enfin, l'évaluation des résultats de cette politique montre les limites de cette évaluation dans un cadre interne à l'agence car les causes de succès ou de réussite sont en partie hors de l'agence. Il nous semble que l'explicitation des options et leur éventuelle substituabilité ou interdépendance est essentielle pour que les responsabilités soient assumées. Dans ces responsabilités, celle relative à la mesure des données du milieu est essentielle car elle rend visible ou non les impacts des actions des différents acteurs.

\section{VI — REMERCIEMENTS}

Ce travail a bénéficié d'une subvention de l'Agence de l'Eau Seine Normandie.

\section{VII $\square$ REFERENCES}

[1] IfEN (2001). - Eau potable : diversité des services... grand écart des prix. Données de l'environnement $\mathbf{n}^{\mathbf{0}} 65$ : 4 pages.

[2] Barraqué, B. (1999). - Assessing the efficiency of economic instruments: the case of the French Agences de l'Eau. Semana Internacional de Estudos sobre Gestão de Recursos Hidricos, Foz do Iguaçu.

[3] Martin, Y. (1998). — Quelques réflexions sur l'évolution des agences de bassin. Annales des Mines (juillet-août 1988) : 117119.

[4] Commissariat général au plan (1997). — Rapport au gouvernement. Evaluation du dispositif des Agences de l'eau. Paris.

[5] Bouleau G. (2003). - Acteurs et circuits financiers de l'eau en France. Paris, ENGREF.

[6] Roche, P.-A., J. Lesavre, et al. (2002). — La directive cadre sur 1'eau: perspectives et enjeux pour l'assainissement des collectivités. $81^{e}$ congrès AGHTM, Montpellier.

[7] Roche P.-A., G. Billen, et al. (2005) Les enjeux de recherche liés à la directive cadre européenne sur l'eau. C.R. Géoscience no 337 : 243-267.

[8] Priet, E. (2004). - Les critères de financement de l'agence de l'eau Seine Normandie. Nanterre, Université Paris X Nanterre et ENGREF : 85 pages.

[9] Guilbert, S., R. Puechberty, et al. (2005). — Eléments d'évaluation de la politique «points noirs» de l'Agence de l'Eau Seine-Normandie. Paris, Agence de l'Eau Seine Normandie: 59 pages. 